\title{
Just the Facts: Risk stratifying non-traumatic back pain for spinal epidural abscess in the emergency department
}

\author{
Zoe Polsky, MD*; Shawn K. Dowling ${ }^{\circledR}, \mathrm{MD}^{\dagger}$; W. Bradley Jacobs, MD ${ }^{\ddagger}$ \\ CLINICAL SCENARIO
}

A 65-year-old male with a history of hypertension presents to the emergency department (ED) with new onset of nontraumatic back pain. The patient is investigated for life-threatening diagnoses and screened for "red flag symptoms," including fever, neurologic abnormalities, bowel/bladder symptoms, and a history of injectiondrug use (IVDU). The patient is treated symptomatically and discharged home but represents to the ED three additional times, each time with new and progressive symptoms. At the time of admission, he is unable to ambulate, has perineal anesthesia, and $500 \mathrm{cc}$ of urinary retention. Whole spine magnetic resonance imaging (MRI) confirms a thoracic spinal epidural abscess. This case, and many like it, prompts the questions: when should emergency physicians consider the diagnosis of a spinal epidural abscess, and what is the appropriate evaluation of these patients in the ED? (Figure 1).

\section{KEY CLINICAL QUESTIONS}

1. When should I consider the diagnosis of a spinal epidural abscess when assessing a patient with non-traumatic back pain?

With previous studies failing to differentiate correlative from causative risk factors for a spinal epidural abscess, the emergency physician must employ a probabilistic approach when considering this diagnosis. ${ }^{1}$ To guide that probability, two specific questions can be asked when considering an infectious etiology for non-traumatic back pain: 1) Is this patient at risk for bacteremia? and 2) Has this patient had direct injection/instrumentation to his spine? ${ }^{1}$ Although a positive response to either question should prompt additional investigations, unfortunately, a negative response to both questions cannot definitively rule out a spinal epidural abscess, as no signs or symptoms can adequately rule out the diagnosis. ${ }^{1}$

While risk factors for infectious spinal pathology, such as IVDU, are well appreciated, the importance of risk factors, including an alternative site of infection, may have previously been overlooked. Therefore, when asking the question of whether the patient is at risk for bacteremia, additional factors should be considered, including recent skin or soft tissue infection, indwelling vascular catheter, fever, conditions resulting in immunosuppression, and history of IVDU. ${ }^{2}$

From the *Department of Emergency Medicine, Cumming School of Medicine, University of Calgary, Foothills Medical Centre, Calgary, AB; ${ }^{\dagger}$ Department of Emergency Medicine, University of Calgary, Rockyview General Hospital - Holy Cross Ambulatory Care Centre, Calgary, AB; and the ${ }^{\ddagger}$ Division of Neurosurgery, University of Calgary, Foothills Medical Centre, Calgary, AB.

Correspondence to: Dr. Zoe Polsky, Department of Emergency Medicine, Cumming School of Medicine, University of Calgary, Foothills Medical Centre, 1403-29 St. NW, Calgary, AB T2N 2T9; Email: zoe.polsky2@ucalgary.ca.

(c) Canadian Association of Emergency Physicians 2020

CJEM 2020;22(6):753-755

DOI 10.1017/cem.2020.422 


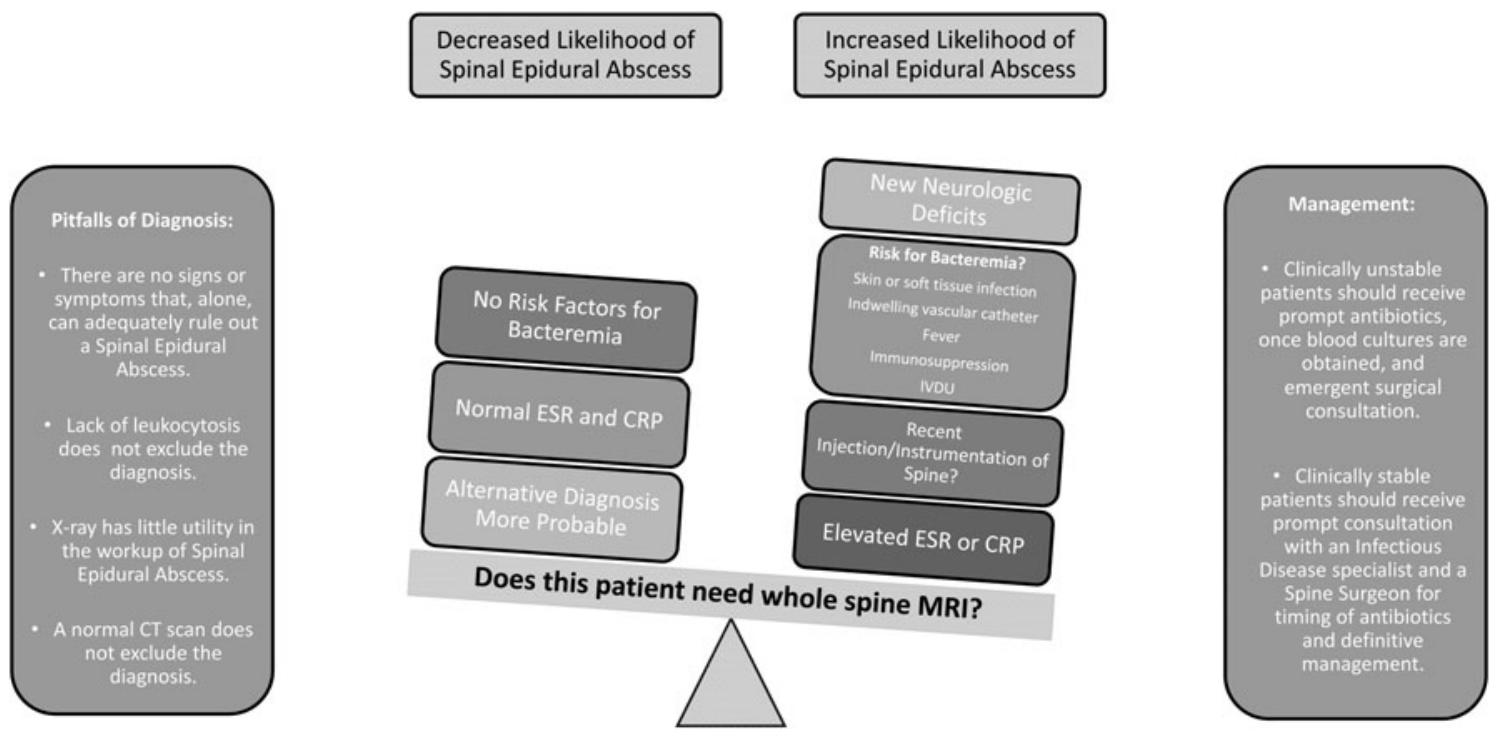

Figure 1. Spinal epidural abscess. With insufficient evidence to support a diagnostic algorithm, the emergency physician must weigh the possibility of this challenging diagnosis.

\section{What lab investigations should be ordered when considering a spinal epidural abscess, and how should results be interpreted?}

Appropriate lab investigations for a spinal epidural abscess include an erythrocyte sedimentation rate (ESR), C-reactive protein (CRP), and blood cultures. A high concordance rate exists between positive blood cultures, and intraoperative abscess cultures, so blood cultures play a crucial role in downstream antibiotic therapy. Erythrocyte sedimentation rate may have superior sensitivity to C-reactive protein for the diagnosis of spinal epidural abscess, with one small single-centre study quoting sensitivities of $100 \%$ and $67 \%$, respectively. ${ }^{3}$ However, due to limited evidence supporting the sole use of erythrocyte sedimentation rate, the combination of both investigations should be ordered, and a whole spine MRI should be obtained if either value is abnormal. Due to the small body of evidence surrounding erythrocyte sedimentation rate and C-reactive protein, if clinical suspicion remains high, despite normal lab values, an MRI should still be pursued. Other laboratory investigations, such as a leukocytosis, lack adequate sensitivity to rule out a spinal epidural abscess. ${ }^{1}$

\section{What is the appropriate imaging for a suspected spinal epidural abscess?}

In the case of a spinal epidural abscess, a patient's symptoms do not reliably localize to the area of spinal pathology, and thus, whole spine, gadolinium-enhanced MRI remains the gold standard, with a sensitivity and specificity of $>$ $90 \% .{ }^{1}$ It is imperative to appreciate the risk of false negatives, if only targeted imaging of the spine is obtained, as noncontiguous "skip lesions" occur in a small subset of patients with a spinal epidural abscess. ${ }^{4}$ Bacteremic patients, or those with neurologic impairment, should undergo emergent MRI. In centres where MRI is not readily available, assuming that the patient is medically stable with no neurologic deficits, arrangement for MRI within 48-72 hours is recommended. Plain radiographs are of minimal utility, while even a contrast-enhanced computed tomography (CT) lacks sufficient sensitivity to rule out a spinal epidural abscess.

\section{What is the appropriate management for patients with a confirmed spinal epidural abscess?}

Although spinal epidural abscesses may result from a number of different organisms, the most causative is Staphylococcus aureus. Empiric treatment should combine a third or fourth generation cephalosporin, for example, 
ceftriaxone, or piperacillin-tazobactam with vancomycin. ${ }^{5}$ If the patient is unstable or has neurologic deficits, early administration of antibiotics and emergent surgical consultation should be pursued. For stable, neurologically intact patients, early consultation with an infectious disease specialist and a spine surgeon should delineate the timing of antibiotics and definitive management. For those patients, definitive opinion on the need for surgery can be obtained within a 48- to 72-hour timeframe. In centres where infectious disease and spinal surgery specialists are not available, a phone consultation should be pursued. All patients with a confirmed spinal epidural abscess should be admitted, to facilitate treatment and close monitoring.

\section{KEY POINTS}

- Current evidence cannot unequivocally delineate a diagnostic algorithm for the workup of a spinal epidural abscess; therefore, a probabilistic approach should be used.

- The first question that an ED physician should consider is whether a patient at risk for bacteremia (recent skin or soft tissue infection, indwelling vascular catheter, fever, conditions resulting in immunosuppression, or history of IVDU).

- The second question for the ED physician to consider is whether the patient had direct injection/instrumentation to his or her spine.

- Lab investigations should include blood cultures, CRP/ESR, and in those patients where a spinal epidural abscess is still being considered, whole spine MRI is the only definitive means of imaging.

- In clinically stable patients without neurologic deficits, timing of antibiotic therapy and need for surgery should be guided in consultation with an ID physician and a spine surgeon.

\section{CASE RESOLUTION}

At the time of admission, the patient underwent surgical decompression for his spinal epidural abscess, followed by a multi-week course of antibiotics. Following his prolonged admission to hospital, the patient continued to progress with physiotherapy and was able to regain strength and ambulate independently.

Keywords: Emergency department, non-traumatic back pain, spinal epidural abscess

Competing interests: WBJ discloses consulting agreements with Stryker, Medtronic, and DePuy-Synthes.

\section{REFERENCES}

1. Bond A, Manian FA. Spinal epidural abscess: a review with special emphasis on earlier diagnosis. Biomed Res Int 2016;epub, 1614328.

2. Davis WT, April MD, Mehta S, Long B, Shroyer S. High risk clinical characteristics for pyogenic spinal infection in acute neck or back pain: prospective cohort study. Am $\mathcal{F}$ Emerg Med 2020;38(3):491-6.

3. Davis DP, Salazar A, Chan TC, Vilke GM. Prospective evaluation of a clinical decision guideline to diagnose spinal epidural abscess in patients who present to the emergency department with spine pain. 7 Neurosurg Spine 2011;14(6):765-70.

4. Ju KL, Do Kim S, Melikian R, Bono CM, Harris MB. Predicting patients with concurrent noncontiguous spinal epidural abscess lesions. Spine 7 2015;15(1):95-101.

5. Berbari EF, Kanj SS, Kowalski TJ, et al. 2015 Infectious Diseases Society of America (IDSA) clinical practice guidelines for the diagnosis and treatment of native vertebral osteomyelitis in adults. Clin Infect Dis 2015;61(6):e26-46. 